\title{
RIVALLAIN, Josette \& IroKo, Félix A. - Yoruba. \\ Masques et rituels africains. Paris, Hazan, 2000, 150 p.
}

Joël Noret

\section{OpenEdition}

\section{Journals}

Édition électronique

URL : http://journals.openedition.org/etudesafricaines/4986

DOI : 10.4000/etudesafricaines.4986

ISSN : 1777-5353

\section{Éditeur}

Éditions de l'EHESS

\section{Édition imprimée}

Date de publication : 1 janvier 2005

ISBN : 978-2-7132-2047-0

ISSN : 0008-0055

\section{Référence électronique}

Joël Noret, «Rivallain, Josette \& IRoko, Félix A. - Yoruba. Masques et rituels africains. Paris, Hazan, 2000, 150 p. », Cahiers d'études africaines [En ligne], 177| 2005, mis en ligne le 11 avril 2005, consulté le 24 septembre 2020. URL : http://journals.openedition.org/etudesafricaines/4986 ; DOI : https:// doi.org/10.4000/etudesafricaines.4986

Ce document a été généré automatiquement le 24 septembre 2020

(c) Cahiers d'Études africaines 


\title{
Rivallain, Josette \& IROKO, Félix A. - Yoruba. Masques et rituels africains. Paris, Hazan, 2000, 150 p.
}

\author{
Joël Noret
}

1 Cet ouvrage de J. Rivallain (maître de conférences au Musée de l'Homme et au Muséum national d'histoire naturelle) et F. A. Iroko (professeur d'histoire à l'Université d'Abomey-Calavi, au Sud-Bénin), abondamment illustré, part d'une intention louable, celle d'introduire à l'importance de certaines sociétés de masques dans les sociétés connues aujourd'hui comme "yoruba». Rappelant l'importance démographique et historique des groupes yoruba en Afrique de l'Ouest, les auteurs évoquent d'abord quelques généralités sur le fonctionnement de leurs sociétés de masques, pour centrer ensuite leur propos sur deux d'entre elles : égun (les célèbres masques de revenants) et gèlèdè (une société de masques jadis principalement destinée à combattre la sorcellerie). Mais dès les premières pages, on se sent mal à l'aise avec certaines propositions des auteurs, qui affirment par exemple, lorsqu'ils évoquent « la ville mère d'Ilé Ifé », que " les Yoruba croient que le premier homme [y] est apparu » (p. 12) et « continuent de lui [Ilé Ifé] rendre hommage dans leurs prières » (p. 15). À l'heure où les villes du Sud-Ouest du Nigeria sont, par exemple, l'un des plus grands foyers d'expansion du pentecôtisme, propageant même celui-ci dans les autres pays d'Afrique, et où, dans ces villes, l'islam y connaît également un succès important, on se demande ce qui peut pousser à tenir ce type de discours sur des "croyances yoruba ", sinon la méconnaissance du terrain ou le désir de prolonger une forme d'exotisme douteuse et un peu suspecte.

2 De façon très rapide, les auteurs relèvent le développement de la dimension " profane " des masques (sans véritable analyse des rapports de pouvoir dans lesquels s'inscrit ce phénomène), la variabilité existant dans l'organisation des sociétés d'initiés, la place discrète des femmes en leur sein (semblant même reprendre à leur compte sans les remettre en perspective des accusations de sorcellerie pesant sur certaines de ces femmes [p. 29]), ainsi que le rôle dévolu aux oracles et le développement de pratiques 
ésotériques qu'on peut y observer (ce que les auteurs nomment «convergence de préoccupations divines, sacrées et occultes »[p. 35]).

3 Les très riches illustrations de l'ouvrage ne suffisent malheureusement pas à compenser la pauvreté des analyses, d'autant plus que celles-ci sont couplées à toute une série d'imprécisions et d'erreurs factuelles. Par exemple, les auteurs croient pouvoir mentionner l'existence d'un revenant dénommé Carlos à Ouidah (Sud-Bénin [p. 25]), qui illustrerait le fait que tous les revenants (égun) ne se voient pas attribuer un nom yoruba (p. 88), mais il n'y a en fait pas de revenant de ce nom à Ouidah, et le Carlos mentionné est un ancien grand initié (aujourd'hui décédé) qui a été étroitement associé à l'un des masques les plus importants de la ville et dont l'histoire est rapportée dans certains cercles d'initiés. Ou encore, l'affirmation selon laquelle les masques égun " ne représentent que des revenants de sexe masculin» (p.26), qui, simplificatrice, n'est que partiellement fausse (dans la mesure où les revenants féminins ne sortent effectivement que très rarement), mais qui se voit contredite par la légende d'une photo (p. 90) montrant la danse d'un masque présenté (probablement abusivement d'ailleurs dans ce cas-là) comme "égun femelle » (voir aussi les pp. 94-95). Un peu plus loin, on peut encore lire que « la divinité par excellence des sociétés égun est agan. Elle est particulièrement puissante et vénérée à Imèko, au Nigeria, à Kétu, Porto-Novo, Ouidah, Sakété, Iyoko, Itako, au Bénin, et à Lomé, au Togo » (p. 32). Or, cette affirmation généralise abusivement (un phénomène qu'on retrouve plusieurs fois dans l'ouvrage, mais dont l'énumération des cas serait fastidieuse), mais aussi déshistoricise (ce qui est plus surprenant dans la mesure où $\mathrm{F}$. A. Iroko est précisément historien et que ce phénomène revient aussi dans l'ouvrage) ce que peut être le culte d'agan (qui est en fait, au Sud-Bénin, entre les mains d'un groupe restreint de dignitaires au sein même de la société initiatique). En effet, pour n'évoquer que quelques-unes des localités mentionnées par les auteurs, Imèko est depuis une trentaine d'années la "ville sainte " de l'Église du Christianisme Céleste (l'une des plus importantes Églises prophétiques d'Afrique de l'Ouest), et l'Agan ne saurait dès lors y être particulièrement puissant. À Ouidah, les cérémonies d'agan n'ont eu lieu que trois fois, et dans un seul quartier, dans les années 1980 et 1990. À Takon ou Itako, l'Agan n'existe que depuis une trentaine d'années, et le culte égun lui-même ne remonte qu'à quatre-vingts ans au maximum. À Lomé, les Yoruba ne forment qu'une toute petite minorité, et l'Agan ne saurait dès lors y être "particulièrement puissant et vénéré ». Pour prendre un dernier exemple concernant cette fois les masques gèlèdè, les auteurs affirment un peu plus loin qu'il est interdit au porteur d'un tel masque de "découvrir son visage» (p.43), avant de présenter eux-mêmes (p. 73) des photos de porteurs au visage semi-découvert et bien reconnaissable pendant leur performance. À un rythme soutenu, J. Rivallain et F. A. Iroko accumulent ainsi imprécisions et erreurs ethnographiques tout au long de l'ouvrage, jusqu'à commenter (pp.120-121) une photo présentant des crânes placés dans des jarres de terre cuite ornées d'étoffes lors d'une cérémonie d'ancestralisation (des populations gun) à Porto-Novo ${ }^{1}$ en soutenant qu'elle montre des masques égun !

Un chapitre est consacré chaque fois aux cérémonies gèlèdè (chapitre III) puis égun (chapitre IV). C'est tout d'abord l'occasion pour les auteurs de passer en revue d'une part les différentes étapes de l'organisation d'une sortie de masques gèlèdè et de leur prestation, d'autre part les contextes dans lesquels les gèlèdè peuvent se produire, et enfin les différentes phases de la fabrication d'un masque. Puis, dans le chapitre consacré aux cérémonies des masques égun, les auteurs soulignent rapidement la variabilité du phénomène avant de généraliser paradoxalement à toute une série de 
niveaux la situation telle qu'ils ont pu l'observer sur leur principal lieu de contact avec le « terrain », à savoir (selon toute vraisemblance) certaines agglomérations du Sud-Est du Bénin.

5 Les auteurs reviennent ensuite dans un cinquième chapitre sur les «influences yoruba » et la propagation des cultes « yoruba » dans le Sud-Est de l'Afrique de l'Ouest et dans les "Amériques noires" suite, en particulier, aux mouvements et aux déplacements de population (et de cultes) liés au commerce de traite, sans jamais cependant problématiser véritablement la catégorie "yoruba", ce qui mène une fois encore à certaines confusions et à une occultation complète de la problématique du traditionalisme et du caractère construit, sinon inventé, des traditions.

6 Dans un sixième chapitre, fort court par ailleurs, les auteurs entendent présenter la dynamique de «banalisation et profanation » des masques, ce qui les fait conclure à un déclin des sociétés de masques sans mentionner le fait pourtant important que la perte partielle de leur caractère secret s'accompagne, au Sud-Bénin, d'une croissance extraordinaire du nombre de "couvents » de masques égun, si bien que ce culte y est aujourd'hui indiscutablement le culte " païen » le plus populaire.

7 Dans un dernier chapitre enfin, J. Rivallain et F. A. Iroko concluent en esquissant l'histoire de la collection par les Européens et de la mise en musée des masques yoruba, histoire qui se limite en fait à l'histoire des collections muséales françaises.

\section{NOTES}

1. Photo à comparer avec celle présentée par M.-J. Jamous dans son article de 1994 « Fixer le nom de l'ancêtre (Porto-Novo, Bénin) », Systèmes de pensée en Afrique noire, 13, pp. 121-157. La photo des jarres en terre cuite contenant les crânes et ornées d'étoffes se trouve à la page 137 . 